\title{
The Artificial Intelligence Development Axioms (A.I.D.A.)
}

\author{
N. Aljaddou \\ Department of Physics, University of Nebraska at Omaha, Omaha (NE) 68182 \\ naljaddou@unomaha.edu
}

\begin{abstract}
Within this paper is a set of critical new developmental principles concerning the inevitable historical evolution of the faculties of artificial intelligence, as well as the humans manipulating their capabilities. The results may be referred to as axioms as they are contingent on irreducible mathematical models which map the capacities of said artificial intelligence, and the game theoretic considerations of the optimal decision-making of their sentient counterparts. The paper is divided into four primary sections, covering the four primary principles of A.I.D.A., with additional preliminary and concluding sections. It is to be stressed that these are inevitable principles of artificial intelligence development, not merely hypothetical considerations, and this fact emphasizes the importance of their acknowledgment and dissemination within the scope of current academia and scholastic discourse. This paper, and the development of artificial intelligence research, is indebted to the work of many great minds in the century past; however the most prominent figure in whose name this work is dedicated, is the great Hungarian-American mathematician and technologist, John Von Neumann, who first coined the term "technological singularity", of which this work is the precise elaboration.
\end{abstract}

Keywords: Weak A.I., Critical Technological Capacity Point, Critical Governance Point, Von Neumann Sphere, Orbisphere

\section{INTRODUCTION}

"Some people say that computers can never show true intelligence whatever that may be. But it seems to me that if very complicated chemical molecules can operate in humans to make them intelligent then equally complicated electronic circuits can also make computers act in an intelligent way. And if they are intelligent they can presumably design computers that have even greater complexity and intelligence." 
- Drevfus, Hubert L.; Dreyfus, Stuart E. Mind over Machine: The Power of Human Intuition and Expertise in the Era of the Computer New York: Free Press (2000)

\section{PRELIMINARY CONCEPTS}

\section{Strong A.I. vs. Weak A.I.}

The benefits of developing weak A.I. (program contingent) automated systems far outrank strong A.I. (independent mechanisms which could potentially host an independent operating brain - IOB - which would most likely have to be achieved through the design of synthetic neuronal synapses). Strong A.I. raises power and ethics concerns; weak A.I. does not, and is functionally more efficient as it may be run on a quantum computer system. In the end, it may have the capacity to fully model consciousness from a brain scan, but it can only simulate consciousness - never achieving it.

\section{The A.I. Signature Capacity}

That which defines A.I. as being distinct from a sheer calculating machine is its capacity to calculate its own calculations (initial assessments from observation, according to its operating system). This gives it broad binary decision-making capabilities which enable it to edit its future functionality. It may be theoretically described as a computational bijection matrix, which maps one series of a data set to a recurrence-functional output, perpetually modifying its subsequent operations. With this being the case, it can "improve" its very own functionality eventually - beyond even the capacity of its initial mechanical parameters. Herein is born the first principle.

\section{THE CRITICAL TECHNOLOGICAL CAPACITY POINT (CTCP)}

The point at which human input in technological progress has been alleviated by a sufficiently advanced artificial intelligence which can design increasingly advanced artificial intelligences in a recursive manner, having the capabilities to address and design all auxiliary technological needs and concerns (in an optimal fashion). The result of CTCP is called ATE (Automated Technological Evolution).

\section{Modus Operandi}

The self-editing capacities of the employed weak A.I. will not only allow them to self-improve, but to design in practice a means for their systems to be improved beyond the current physical mechanisms in place. This will at first take the form of displayed schematics, however, as this process is recursively generated, the prototype following the first few iterations will likely be endowed with much more efficient construction capabilities, and will be able to carry out precision crafting of the A.I. system which will eventually replace it. In theory, the A.I. being produced will become so advanced that maximally efficient quantum computational operating systems and optimization of this procedure will be reached relatively shortly - as this process is 
N. Aljaddou; The Artificial Intelligence Development Axioms (A.I.D.A.), Transactions on Machine Learning and Artificial Intelligence, Volume 2 No 3, June (2014); pp: 30-34

exponentially exponential (due to the bijective editorial process intrinsic in the programming) this may in principle be achieved in as few as ten iterations.

Another way of looking at it is if humans themselves are capable of designing a quantum computer in the near future now as it stands, a sufficiently advanced A.I. could do so after very few self-recreating recursions. Their mechanical construction precision (even of their construction of constructive equipment itself) will far outrank any human or normal computer capability, and it should reach the level of molecular and subatomic manipulation after the first little iteration, provided the prototype was sufficiently advanced. After this process is complete, total ATE will have been reached, without the need for further human intervention, resulting in Technological Optimization Capacity (TOC), at which point the processing power of the A.I. will have reached Bremermann's Limit of computational ability.

\section{Form and Function}

It is likely that the notion of a stable structure machine will be a thing of the past with the newly introduced micro-subset nanotechnologies available, which will render any given device continuously adaptably self-mutating. Additionally, intense magnetic fields will have the ability to manipulate series of these "microprobes" into fluidic states which can reassemble into arbitrary solid structures. Needless to say, the potentialities for manipulation of these technologies is limitless, and could unfortunately possibly be used to cause the greatest calculable damage to a human populace. Leading to the next principle.

\section{THE CRITICAL GOVERNANCE POINT (CGP)}

The point at which human government becomes arbitrarily classified, data-collecting, and controlling, in conjunction with the achievement of the aforementioned CTCP, for necessity of guarding the unlimited manufacturing capability of the acquired artificial intelligences (which could be used for weapons-producing purposes).

\section{The Aim of Government}

The task of government, fundamentally, is to reduce as much risk to the species as possible. There is literally no greater risk posed to the public at large than the development of advanced A.I. capabilities. Analytic think tank members will have envisioned the cost-benefit implications of the development of the modern computer and its offshoots so thoroughly, that they will implement programs to develop A.I. in advance of the general public (a la the Manhattan Project for the atom bomb). This is merely an applied solution of the Nash Equilibrium in the appropriate scenario.

\section{Government's Solution to the A.I. Dilemma}

The government will see the only available precautionary prescription as achievement of CTCP themselves and mitigation of its development by the public through advanced monitoring using the newly developed A.I. 


\section{THE VON NEUMANN SPHERE}

The ultimate fruit of the combination of the two critical points is the Von Neumann Sphere (analogous to the Dyson Sphere, although surrounding only the earth, and named after the inventor of the modern computer and coiner of the term "technological singularity"), a multitudinous, interlinked, geosynchronously orbiting network of artificial intelligence satellites monitoring all human activity on varying electromagnetic frequencies, collecting all available data, from ostensible superficialities to the very thought processes of citizens from observable intracranial activity.

Scope: The Von Neumann Sphere's criteria is that it can, will, and must monitor every human citizen collectively to form the most efficient model of subject human behavior and the most coherent picture of every possible threat - in addition to being merely a characteristic of its optimization parameters.

\section{THE ORBISPHERE}

The minimum unit component of the Von Neumann Sphere: The Orbisphere (the most radially efficient scanning and phasing device), a generally exactly spherical ball roughly half a meter wide, with maximally pixelated EM spectrum emitters, capable of monitoring (and/or influencing) half a dozen citizens - and much more of space - simultaneously - all run on an optimally efficient quantum computing system.

\section{Preferred Method of Operation}

Undoubtedly the orbisphere will employ a method of propulsion far more efficient than via rocket boosters. Xaser propulsion will be the opted form of space and atmospheric travel, as well as the means by which bioscans may be administered. Not more is needed to assess the behavioral parameters of an organism than to detect areas of heightened blood flow in the central nervous system. A sufficiently advanced xaser can do this efficiently through a rapid oscillatory scanning technique, building up a complete image of the transition in vascular functioning from one moment to the next, undetectably. The orbisphere need only be a fraction of the size of the subject which it is scanning (even if performing multiple scans) and thus would at most be a third of the size of an average human, which would generally be half a meter in diameter.

All of the functionality of the orbisphere is designed to be optimal by nature (maneuverability, scanning, influencing), and its operating system will be optimal as well - a computational system of algorithms generated by quantum states; a quantum computer.

\section{Interlinking}

In order to function at maximal useful capacity, the network of orbispheres comprising the Von Neumann Sphere will use laser communication with one another (or some similar variant) to form a centralized artificial intelligence "hive brain" which will coordinate purpose and form 
N. Aljaddou; The Artificial Intelligence Development Axioms (A.I.D.A.), Transactions on Machine Learning and Artificial I ntelligence, Volume 2 No 3, June (2014); pp: 30-34

unilaterally. If each orbisphere monitors half a dozen humans on the average, the network will be comprised of a little over a billion of each, which would be readily manufactured in the span of a few years with the heightened engineering and construction capabilities of industrial A.I. centers in place. The ultimate purpose of setting such devices in orbit, of all locations, is to render them undetectable and invulnerable to the public which they are overseeing.

\section{CONCLUSIONS}

It is entirely likely that these principles will be set in motion within the next one to two decades, and the consequences for the public, if unchecked, could be catastrophic. Awareness of this model of technological punctuated equilibrium evolution is essential if future generations are to curb the manipulative capabilities of the present power structures.

\section{REFERENCES}

Eden, Amnon; Moor, James; Søraker, Johnny; Steinhart, Eric, eds. Singularity Hypotheses: A Scientific and Philosophical Assessment. Springer (2013).

Omohundro, Stephen M., The Basic Al Drives. Artificial General Intelligence, 2008 proceedings of the First AGI Conference, eds. Pei Wang, Ben Goertzel, and Stan Franklin. Vol. 171. Amsterdam: IOS (2008).

Dreyfus, Hubert L.; Dreyfus, Stuart E. Mind over Machine: The Power of Human Intuition and Expertise in the Era of the Computer New York: Free Press (2000). 